\title{
Human papillomavirus infection and expression of ATPase family AAA domain containing 3A, a novel anti-autophagy factor, in uterine cervical cancer
}

\author{
TAI-CHANG CHEN ${ }^{1,2}$, YAO-CHING HUNG ${ }^{3,4}$, TZE-YI LIN ${ }^{5}$, HUI-WEN CHANG ${ }^{5}$, \\ I-PING CHIANG ${ }^{5}$, YUEH-YING $\mathrm{CHEN}^{6}$ and $\mathrm{KUAN}^{-\mathrm{CHIH}} \mathrm{CHOW}^{7}$
}

\begin{abstract}
${ }^{1}$ Department of Obstetrics and Gynecology, National Taiwan University Hospital Hsin Chu Branch, Hsin Chu; ${ }^{2}$ Department of Industrial Management, Chung Hua University, Hsin Chu; ${ }^{3}$ College of Medicine, China Medical University, Taichung; ${ }^{4}$ Department of Obstetrics and Gynecology, China Medical University Hospital, Taichung; ${ }^{5}$ Department of Pathology, China Medical University Hospital, China Medical University, Taichung; ${ }^{6}$ Department of Laboratory Medicine, Taipei Veterans General Hospital Su-Ao Branch, I-Lan; ${ }^{7}$ Graduate Institute of Biomedical Sciences, National Chung Hsing University, Taichung, Taiwan, R.O.C.
\end{abstract}

Received May 4, 2011; Accepted June 10, 2011

DOI: 10.3892/ijmm.2011.743

\begin{abstract}
Our aim was to determine the association of human papillomavirus (HPV) infection with the expression of ATPase family AAA domain containing 3A (ATAD3A), an anti-autophagy factor, in uterine cervical cancer (UCC). The HPV genotype was determined by an Easychip HPV blot assay. ATAD3A expression was determined by immunohistochemical staining. High-risk HPV (hrHPV) was detected in $184(88.9 \%)$ of 207 UCC cases. ATAD3A expression was detected in 164 (79.2\%) UCC cases. A significant correlation was found between ATAD3A expression and the presence of hrHPV $(p<0.001)$, FIGO stage $(\mathrm{p}=0.014)$, lymph node involvement $(\mathrm{p}=0.001)$, c-MET expression $(\mathrm{p}<0.001)$, interleukin- 8 $(\mathrm{p}=0.03)$ and patient survival $(\mathrm{p}=0.0016)$. Interestingly, silencing of E6/E7 expression decreased ATAD3A expression and cell survival. Moreover, knockdown of ATAD3A $\left(\right.$ ATAD $3 A^{\mathrm{kd}}$ ) expression or addition of resveratrol, increased cellular autophagy and apoptosis and reduced drug resistance. Resveratrol reduced ATAD3A expression, and increased abrasion of the mitochondrial outer membrane as well as numbers of autophagosomes, the phenomena that were frequently found in ATAD3A ${ }^{\mathrm{kd}}$ cells. In conclusion, our results show that
\end{abstract}

Correspondence to: Dr Kuan-Chih Chow, Graduate Institute of Biomedical Sciences, National Chung Hsing University, Taichung 40227, Taiwan, R.O.C.

E-mail: kcchow@dragon.nchu.edu.tw

Abbreviations: HPV, human papillomavirus; hrHPV, high-risk HPV; ATAD3A, ATPase family AAA domain containing 3A; UCC, uterine cervical cancer

Key words: high risk HPV, ATAD3A, drug resistance, autophagy, prognosis
HPV infection correlates with increased ATAD3A expression and drug resistance in UCC. Persistent HPV infection may stabilize ATAD3A expression to inhibit cell autophagy and apoptosis as well as to increase drug resistance.

\section{Introduction}

Uterine cervical cancer (UCC) is the most prevalent cancer, and the fifth leading cause of cancer-related deaths in Taiwanese women (1). High incidence and mortality of the disease was in part due to the conservative attitude toward the Papanicolaou smear, because even though the disease could be detected at the early stages the method was considered intrusive, and in part due to the high infection rate of human papillomavirus (HPV) among the diseased women (2).

HPV infection is a potential etiologic cause for UCC development $(3,4)$. Among 95 known HPV (5), genotypes 16, 18,52 and 58 are categorized as high-risk and most frequently detected in Taiwanese UCC patients (6-9). Carcinogenicity of the high-risk HPV (hrHRV) is closely associated with E6 and E7 proteins, which can respectively inhibit activities of tumor suppressors, p53 and retinoblastoma protein (pRB), to interrupt cell growth regulation (10). Besides carcinogenesis, persistent HPV infection further correlates with tumor recurrence, cancer cell radiosensitivity and the resulting poor prognosis (11-13).

Oncogene activation is also crucial for tumorigenesis and cancer progression; in particular, genes that are associated with disease advancement and, possibly, resistance to irradiation and anticancer chemotherapeutics. By using differential display, we have identified overexpression of dihydrodiol dehydrogenase (DDH) in non-small cell lung cancer (14). Using microarrays, we further found that DDH was overexpressed in cisplatin-resistant ovarian cancer cells (15). Clinically, patients with elevated DDH have a higher tumor recurrence rate and lymph node metastasis, and the tumor is more resistant to cisplatin-based chemotherapy (16). 
Interestingly, DDH overexpression has also been identified in UCC (6). Nevertheless, since DDH does not directly inactivate anticancer chemotherapeutics, the pro-tumorigenic effects are yet to be clarified. Recently, using suppression subtractive hybridization, oligonucleotide microarray, hierarchical clustering and subsequent gene pool analysis, we identified several interesting genes, such as dynamin-related protein 1 (DRP1) (17), mitofusin 2 (Mfn2) and ATPase family, AAA domain containing 3A (ATAD3A) (18), which were associated with drug resistance of lung cancer cells. DRP1 and Mfn2 are GTPases, and ATAD3A is an ATPase. Interestingly, serum deprivation increased protein levels of ATAD3A and cisplatin resistance. Silencing of ATAD3A expression, however, increased cisplatin sensitivity, mitochondrial fragmentation and cellular autophagy, suggesting that ATAD3A could be an anti-apoptotic factor, essential for maintaining the mitochondrial integrity (18). However, the role of ATAD3A expression in UCC has not been investigated.

In this study, we determined the genotypes of hrHPV by an Easychip HPV blot assay and ATAD3A expression by an immunohistochemical staining in the UCC specimens. The relationship between the ATAD3A expression and clinicopathological parameters as well as prognostic significance of ATAD3A in UCC patients was statistically evaluated. Correlation of ATAD3A expression and drug resistance was further assessed in the in vitro experiments.

\section{Materials and methods}

Patients and tissue samples. From September 1995 to June 2004, samples from 207 consecutive patients who were diagnosed with UCC were collected. Staging of the disease was performed according to the International Federation of Gynecology and Obstetrics (FIGO) classification. The Medical Ethics Committee approved the protocol, and written informed consent was obtained from every patient before surgery. All patients had undergone surgical resection and radical lymph nodes dissection. Tumor size, lymph node number, differentiation, vascular invasion and mitotic number were also evaluated. Patients with lymph node involvement and those with distant metastasis were treated with chemotherapy (19). Following treatment, all patients were routinely followed every 3-6 months in the Out-Patient Department. Tumor recurrence and metastasis were identified when blood examination, biochemical studies, abdominal sonography, whole body bone scan and the computerized tomography scans of abdomen showed any suspected evidence of the disease. Tissue arrays of UCC specimens from 205 American patients (US Biomax, Inc., Rockville, MD, USA) were used to compare ATAD3A expression between the Chinese and the American patients.

HPV genotyping by Easychip HPV blot assay. Easychip HPV blot assay was used to determine the genotype of HPV infection in pathological sections $(7,8)$. Briefly, DNA extracted from paraffin sections was amplified in a polymerase reaction using HPV-specific MY11/GP6 ${ }^{+}$consensus primers (7). The GP6 ${ }^{+}$ was biotin-labeled at the $5^{\prime}$ end. The amplified DNA fragments were hybridized on an Easychip ${ }^{\circledR}$ HPV blot membrane, which contained 39 genotypes of HPV oligonucleotides (King Car Food Industrial Co., Ltd., Taiwan). The membrane was probed with alkaline phosphatase-conjugated streptavidin, and developed in nitroblue tetrazolium (NBT)/5-bromo-4chloro-3-indolyl phosphate (BCIP) mixture. The purple-blue precipitates were identified as a positive signal.

In situ hybridization. A nonisotopic method, with biotin-labeled DNA probes to HPV 16, 18, 31, 33 and 35 (Dako, Glostrup, Denmark), was used to determine the genotype of HPV in the American sections (6). Cells with dark purple precipitates in nuclei were considered positive for HPV infection.

Immunohistochemical staining and immunoblotting. Immunohistochemical staining was performed on paraffin-embedded tissues by an immunoperoxidase method as previously described $(6,14-18)$. Following removal of paraffin with xylene and alcohol, pathological sections were incubated with antibodies specific to ATAD3A (18), hepatocyte growth factor (HGF), HGF receptor (HGFR, or product of proto-oncogene c-met, c-MET), IL-8 (R\&D Systems, Minneapolis, MN) or Ki-67 (Zymed, San Francisco, CA). The slides were then treated with peroxidase-conjugated secondary antibodies, and developed in 3-amino-9-ethylcarbazole (AEC). The crimson precipitates were identified as positive staining. The non-tumor counterpart of the cervical tissue served as a negative control, and a section of mouse liver tissue was used as positive control for each run of immunohistochemical staining. The same antibodies were used for immunoblotting, which was performed as previously (14-18).

Cell cultures, and drug treatment. In this study, five human cell lines were used, including three squamous cell carcinomas (SKG-I, SKG-II and SKG-IIIa), and two endocervical adenocarcinomas (Nuz and HeLa). Culture media and fetal calf serum (FCS) were from Gibco Laboratories (Grand Island, NY). Other materials were reagent grade from Sigma (St. Louis, MO) and Merck (Darmstadt, Germany). The cells were grown at $37^{\circ} \mathrm{C}$ in RPMI-1640 plus $10 \%$ FCS, and all the media were supplemented with $3 \mathrm{mM}$ glutamine, penicillin $(100 \mathrm{IU} / \mathrm{ml})$ and streptomycin $(100 \mu \mathrm{g} / \mathrm{ml})$.

Drug-sensitivity assay. Drug-sensitivity was measured by a WST-1 assay (20). Cells were seeded at 100, 1,000 and 5,000 cells/96-well plate $18 \mathrm{~h}$ prior to drug challenge. Cells were continuously incubated with various concentrations (ranging from 0.1-50 $\mu \mathrm{M}$ ) of anticancer drugs, e.g., cisplatin, doxorubicin and paclitaxel. The negative control group included cells that were treated with the same concentration of DMSO, a solvent for the drug. Total survival of the cells was determined $72 \mathrm{~h}$ following drug challenge, and percent survival was estimated by dividing optical absorbance resulting from each test group with that of control group. Each experiment was performed in triplicate, and the optical absorbance was measured by coloration of the oxidized substrate, WST-1 (BioVision, Mountain View, CA), which was catalyzed by mitochondrial dehydrogenase.

Inhibition of ATAD3A expression by siRNA-producing lentivirus. ATAD3A expression in SKG-I cells was inhibited by small interference RNA (siRNA) produced by lentivirus infection (18). The infected cells were selected with $1.0 \mu \mathrm{g} / \mathrm{ml}$ 
Table I. Genotype distribution of high-risk HPV infection in patients with uterine cervical cancer.

HPV infection $(n=184)$

\begin{tabular}{|c|c|c|c|c|}
\hline \multirow[t]{2}{*}{ Genotype } & \multicolumn{2}{|c|}{ Single type $(n=158)$} & \multicolumn{2}{|r|}{ Multiple types $(n=26)$} \\
\hline & $\mathrm{n}$ & $\%$ & $\mathrm{n}$ & HPV genotype/genotype (n) \\
\hline \multicolumn{5}{|c|}{ High-risk $\mathrm{HPV}^{+}$} \\
\hline HPV 16 & 76 & 48.1 & 12 & HPV 16/31 (6); HPV 16/35 (1); HPV 16/39 (3); HPV 16/55 (2) \\
\hline HPV 18 & 28 & 17.72 & 1 & HPV $18 / 31(1)$ \\
\hline HPV 31 & 5 & 3.16 & 2 & HPV 31/39 (1); HPV 31/44 (1) \\
\hline HPV 33 & 6 & 3.8 & 1 & HPV 33/52 (1) \\
\hline HPV 52 & 17 & 10.76 & 5 & HPV 52/55 (2); HPV 52/56 (1); HPV 52/61 (2); HPV 52/84 (MM8, 1) \\
\hline HPV 56 & 5 & 3.16 & 1 & HPV 56/70 (1) \\
\hline HPV 58 & 21 & 13.29 & 4 & HPV 58/70 (2); HPV 58/74 (1); HPV 58/67 (1) \\
\hline
\end{tabular}

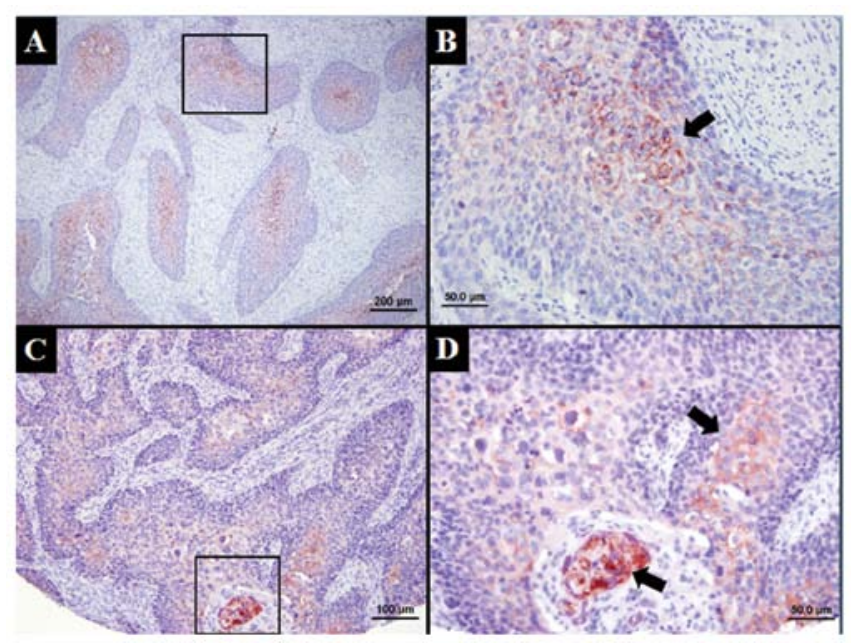

Figure 1. Representative examples of ATAD3A expression in the uterine cervical cancer cells. ATAD3A was detected by immunohistochemistry, and the slides were counterstained with hematoxylin. (A) In uterine cervical cancer, overexpression of ATAD3A was detected in tumor nests (original magnification, x100). (B) Magnification of the Chinese UCC tissue showed that overexpression of ATAD3A was mainly detected in the center of the tumor nest (arrow) (original magnification, x400). (C) In a tissue microarray of American UCC specimens, overexpression of ATAD3A was detected in UCC cells (original magnification, x100). (D) Magnification of the American tissue microarray showed that overexpression of ATAD3A was mainly detected in the center of the tumor nest (arrows) (original magnification, $\mathrm{x} 400$ ).

puromycin for $48 \mathrm{~h}$. ATAD3A expression in cells was examined by immunoblotting.

Slide evaluation. Non-tumor cervical tissue served as internal negative control. Slides were evaluated by two independent pathologists without clinicopathological knowledge. An ImmunoReactive Scoring system was adapted for the study (21). Briefly, a specimen was considered positive if $>25 \%$ of cancer cells were positively stained; or negative, if $<25 \%$ were positively stained.

Statistical analysis. The relationship between ATAD3A expression and clinicopathological parameters was analyzed by the Chi-square test. Survival curves were plotted with the
Kaplan-Meier method (22). Statistical difference of survivals between different groups was compared by the log-rank test (23). Statistical analysis was performed using GraphPad Prism5 statistical software (San Diego, CA). Statistical significance was set at $\mathrm{p}<0.05$.

\section{Results}

High-risk genotype HPV infection and ATAD3A expression. hrHPV, e.g. HPV 16, 18, 31, 33, 52, 56, 58, signal was detected in $184(88.9 \%)$ of 207 specimens, among which 158 $(85.9 \%)$ specimens contained single genotype and $26(14.1 \%)$ contained double genotypes. Low-risk HPV (lrHPV), e.g. HPV 43, 54, 61, 70, was detected in 15 (7.25\%) of 207 specimens. Eight patients were negative for HPV infection. The genotype distribution of hrHPV infection is listed in Table I. Interestingly, ATAD3A (Fig. 1), which was not expressed in non-tumor uterine cervical tissues (NTUCT), was detected in $159(86.4 \%)$ of $\mathrm{hrHPV}^{+}, 3(20 \%)$ of $1 \mathrm{rHPV}^{+}$and $2(25 \%) \mathrm{of} \mathrm{HPV}^{-}$ cases. Among hrHPV cases $(n=23)$, only $5(21.7 \%)$ expressed ATAD3A. ATAD3A expression was positively correlated with hrHPV infection [p $<0.001$, and Spearman correlation (SC), 0.501]. Cell cycle-related Ki-67 was detected in 64 (30.09\%) specimens. Pro-inflammatory or metastasis-related cytokines and receptor, such as, HGF, IL-8 and c-MET, were detected in $72(34.8 \%), 135(65.2 \%)$ and $122(58.9 \%)$ of 207 samples. Correlation of ATAD3A with c-MET was positive $(\mathrm{p}<0.001$; $\mathrm{SC}, 0.299)$. So was that with IL-8 ( $\mathrm{p}=0.03$; SC, 0.151) and Ki-67 ( $\mathrm{p}=0.05$; SC, 0.136). Statistically significant difference, moreover, was found with respect to the FIGO stages $(\mathrm{p}=0.014 ; \mathrm{SC},-0.217)$, mitotic index $(\mathrm{p}<0.001)$ and lymph node involvement $(\mathrm{p}=0.001 ; \mathrm{SC}, 0.225)$. No significant difference was found in the tumor type or expression of HGF. Results are summarized in Table II. ATAD3A overexpression was detected in $142(69.3 \%)$ of 205 American specimens (Fig. 1C and D). Using in situ hybridization (6), HPV signals were detected in $156(76.09 \%)$ of the American specimens. Correlation of HPV infection with ATAD3A expression was positive as well $(\mathrm{p}<0.001 ; \mathrm{SC}, 0.767)$. A statistically significant difference was detected in ATAD3A expression between the American specimens and the Chinese UCC patients $(\mathrm{p}=0.021)$. Tendency 
Table II. Comparison of parameters between patients with and without ATAD3A overexpression.

\begin{tabular}{|c|c|c|c|}
\hline \multirow[b]{2}{*}{ Category } & \multicolumn{2}{|c|}{ ATAD3A expression } & \multirow[b]{2}{*}{ p/Pearson's R } \\
\hline & $\operatorname{High}(n=164)$ & Low $(n=43)$ & \\
\hline \multicolumn{4}{|l|}{ FIGO stage } \\
\hline $\mathrm{Ia}+\mathrm{Ib} 1(\mathrm{n}=86)$ & 60 & 26 & $0.014^{\mathrm{a} /}-0.217$ \\
\hline $\mathrm{Ib} 2(\mathrm{n}=46)$ & 36 & 10 & \\
\hline II $(n=44)$ & 40 & 4 & \\
\hline III $(n=31)$ & 28 & 3 & \\
\hline \multicolumn{4}{|l|}{ Lymph node involvement } \\
\hline Positive $(\mathrm{n}=83)$ & 75 & 8 & $0.001 \% / 0.225$ \\
\hline Negative $(n=124)$ & 89 & 35 & \\
\hline \multicolumn{4}{|l|}{ Tumor type } \\
\hline $\operatorname{SCC}(n=167)$ & 128 & 39 & $0.173^{\mathrm{a} /-0.119}$ \\
\hline Adenocarcinoma $(n=31)$ & 28 & 3 & \\
\hline Mixed $(\mathrm{n}=9)$ & 8 & 1 & \\
\hline Mitotic index (n/10 HPF) & $4.5 \pm 1.8$ & $1.6 \pm 0.9$ & $<0.001^{\mathrm{b}}$ \\
\hline \multicolumn{4}{|l|}{ c-MET } \\
\hline $\operatorname{High}(\mathrm{n}=122)$ & 109 & 13 & $<0.001^{\mathrm{a} / 0.299}$ \\
\hline Low $(n=85)$ & 55 & 30 & \\
\hline \multicolumn{4}{|l|}{ HGF } \\
\hline High $(n=72)$ & 62 & 10 & $0.075^{\mathrm{a}} / 0.124$ \\
\hline Low $(n=135)$ & 102 & 33 & \\
\hline \multicolumn{4}{|l|}{ IL-8 } \\
\hline High $(n=135)$ & 113 & 22 & $0.03 \% / 0.151$ \\
\hline Low $(n=72)$ & 51 & 21 & \\
\hline \multicolumn{4}{|l|}{ Ki-67 } \\
\hline Positive (n=64) & 56 & 8 & $0.05 \% / 0.136$ \\
\hline Negative $(n=143)$ & 108 & 35 & \\
\hline \multicolumn{4}{|l|}{ Oncogenic HPV infection } \\
\hline Positive $(\mathrm{n}=184)$ & 159 & 25 & $<0.001 \% / 0.501$ \\
\hline Negative $(n=23)$ & 5 & 18 & \\
\hline
\end{tabular}

${ }^{a}$ Two-sided p-value determined by the $\chi^{2}$ test. ${ }^{b}$ Two-sided p-value determined by t-test. SCC, squamous cell carcinoma; HPF, high power field.

A

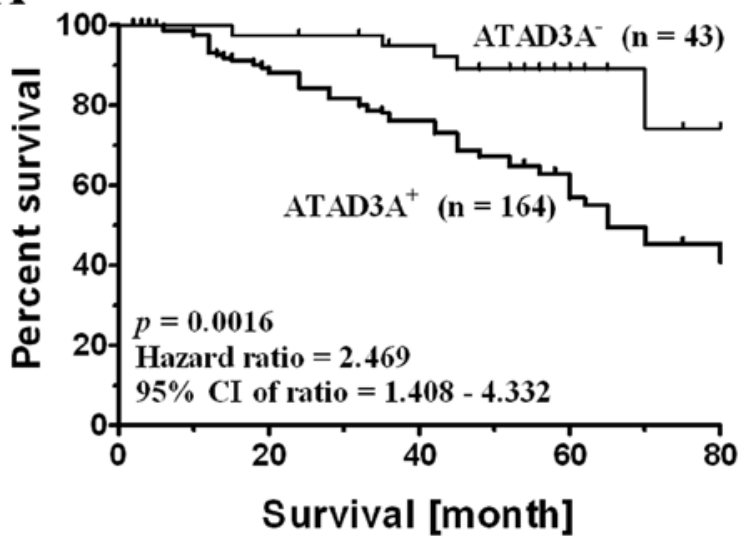

B

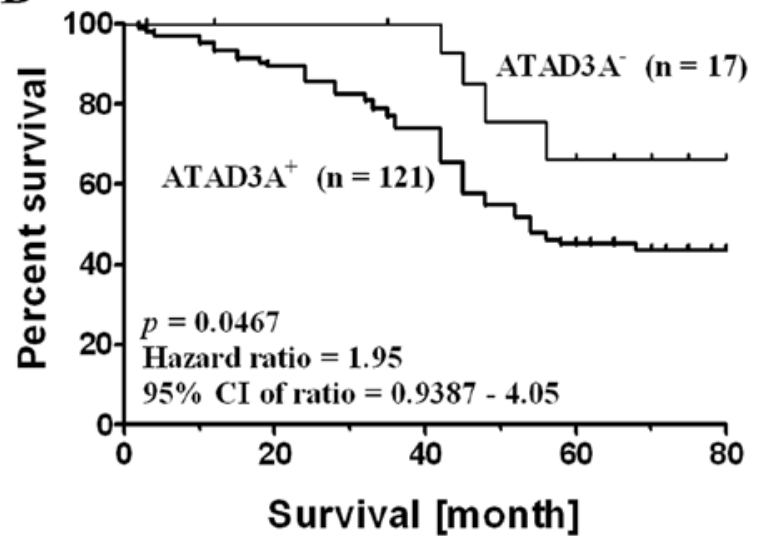

Figure 2. Cumulative survival curves in Chinese UCC patients. Survival curves were plotted with Kaplan-Meier method. Statistical difference of survival between the two groups was compared by a log-rank test. Patient groups were divided according to (A) expression of ATAD3A ( $\mathrm{p}=0.0016)$. (B) Cumulative survival curves of Chinese UCC patients in whom tumor was beyond stage Ib2 and received cisplatin-contained chemoradiotherapy. Patient groups were divided according to expression of ATAD3A $(\mathrm{p}=0.0467)$. 
A

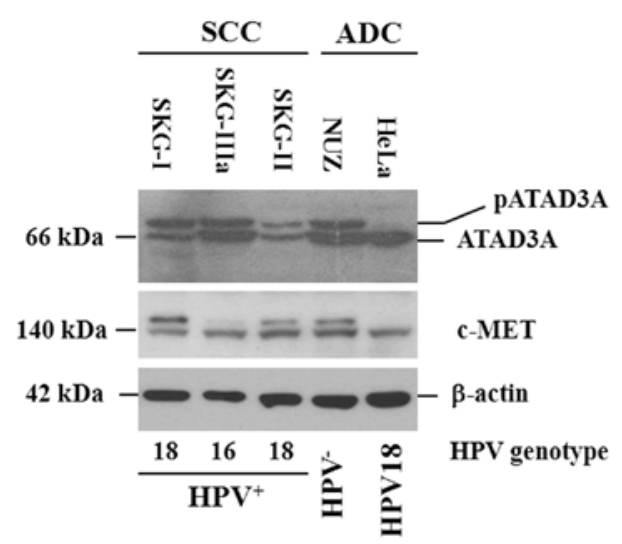

C

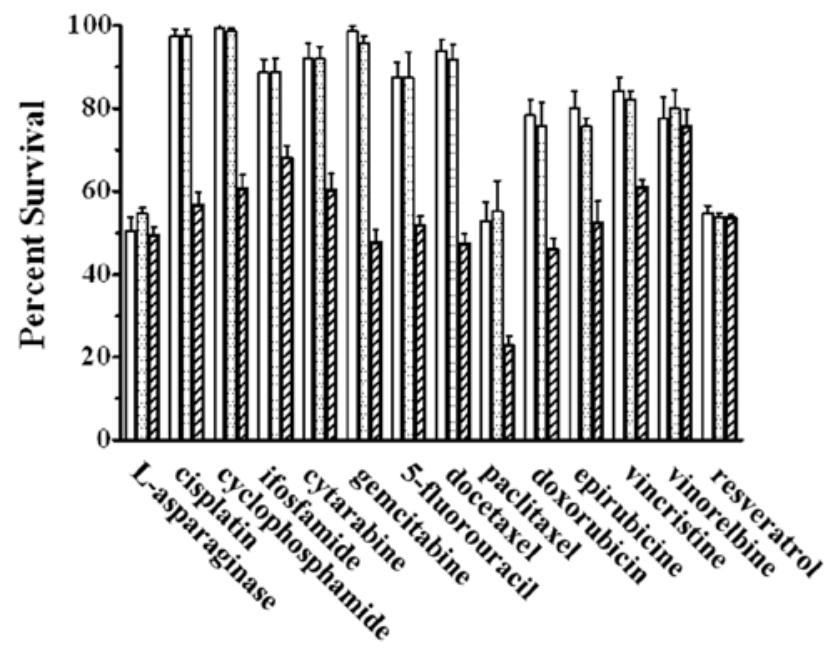

B
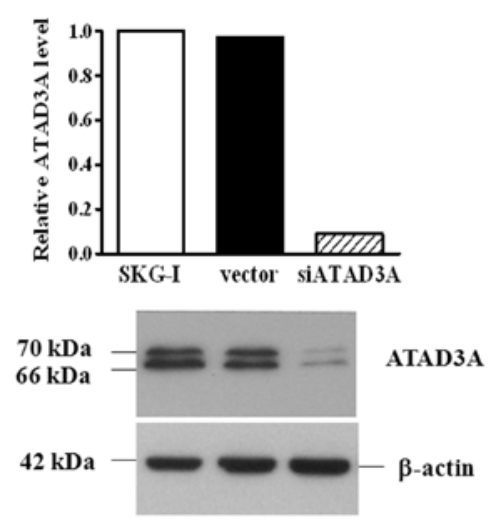

D

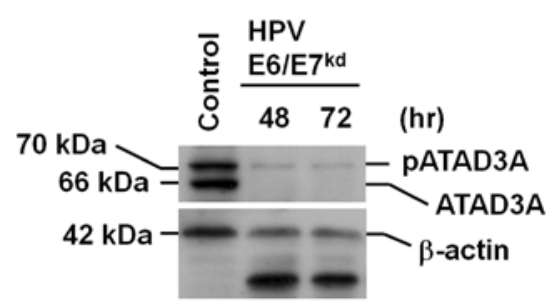

$\mathbf{E}$

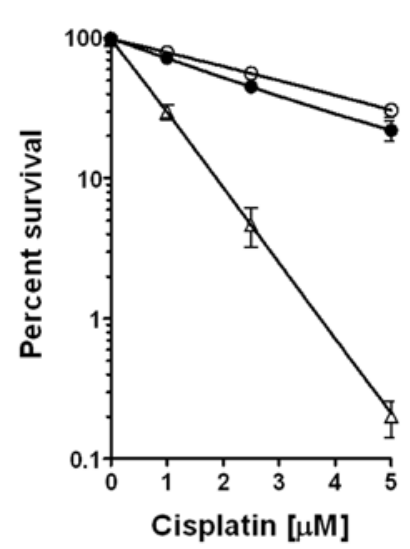

Figure 3. Immunoblot analysis of the expression of ATAD3A in various cancer cell lines derived from cervix. (A) Expression of the 66-kDa ATAD3A was detected in all $3 \mathrm{HPV}$-infected squamous cell cancer (SCC) cell lines by immunoblotting, though the expression levels varied. Expression of c-MET was detected in all 5 cell lines. Interestingly, expression of phosphorylated c-MET (upper band) correlated with that of ATAD3A. (B) SKG-I cells were infected by lentivirus or a virus containing ATAD3A-targeting siRNA-generating hairpin sequence (shATAD3A) to suppress ATAD3A expression. ATAD3A content was verified by quantitative RT-PCR (upper panel) and immunoblotting (lower panel). After cells were infected with ATAD3A-targeting shRNA-lentivirus, ATAD3A expression was decreased about 10-fold in shATAD3A-infected SKG-I cells. (C) Drug sensitivity of SKG-I, vector virus- and shATAD3A-infected cells to anticancer drugs. Percent survival is plotted against a single dose of the drug. Survivals are the mean of triplicate experiments. SKG-I and vector virus-infected cells that had a higher content of ATAD3A were more resistant to anticancer drugs. The difference was significant $(\mathrm{p}<0.001)$. No significant difference was found between SKG-I and vector virus-infected cells ( $\mathrm{p}=0.94)$. White bar, SKG-I; dotted bar, SKG-I infected with vector virus; hatched bar, SKG-I infected with shATAD3A virus. (D) Silencing of E6 and E7 expression by shRNAs (HPV E6/7 ${ }^{\mathrm{kd}}$ ) reduced expression of ATAD3A and $\beta$-actin as determined by immunoblotting. (E) Silencing of E6/E7 increased cisplatin sensitivity in UCC cells. O, SKG-I, wild-type; $\bullet$, SKG-I, E6/E7 ${ }^{\mathrm{kd}}$ with scrambled shRNA; $\triangle$, SKG-I, E6/E7 ${ }^{\mathrm{kd}}$ with shRNA. F-test, $\mathrm{p}<0.01$; No significant difference was found between SKG-I and scrambled shRNA-transfected cells ( $\mathrm{p}=0.91$ ).

to detect overexpression of ATAD3A in Chinese patients was 1.69-fold higher than that in the American specimens.

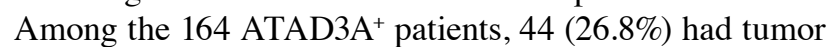
recurrence, and among the $43 \mathrm{ATAD}^{-} \mathrm{A}^{-}$patients, $4(9.3 \%)$ patients had tumor recurrence. The statistical difference of tumor recurrence was significant $(\mathrm{p}=0.008)$. Survival rate of $\mathrm{ATAD} \mathrm{A}^{+}$patients was also significantly worse than that of ATAD3A- patients (Fig. 2A) $(\mathrm{p}=0.0016)$. Using multivariate analysis, FIGO stages, hrHPV infection, lymph node involvement and ATAD3A overexpression were four important factors correlated with poor prognosis for UCC patients. When survival of UCC patients, of whom tumors were above stage
Ib2 and received cisplatin-containing chemoradiotherapy, was analyzed according to ATAD3A expression, the difference was significant as well (Fig. 2B) $(\mathrm{p}=0.0467)$, confirming that ATAD3A overexpression was correlated with drug resistance in UCC cells.

Association of ATAD3A expression and hrHPV infection with drug resistance in cancer cell lines derived from cervix. As determined by immunoblotting, protein levels of ATAD3A were evidently different among various uterine cervical cancer cell lines. Levels of ATAD3A were high in SKG-I, SKG-IIIa, NUZ and HeLa cells, but low in SKG-II cells (Fig. 3A). 
A

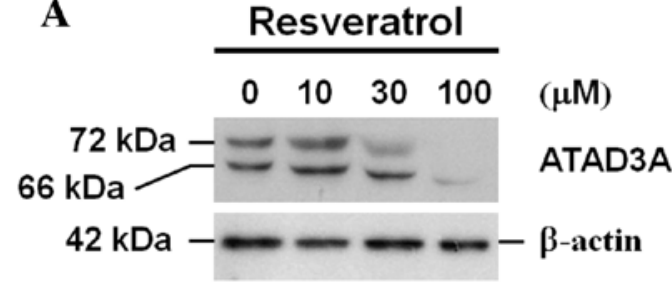

B

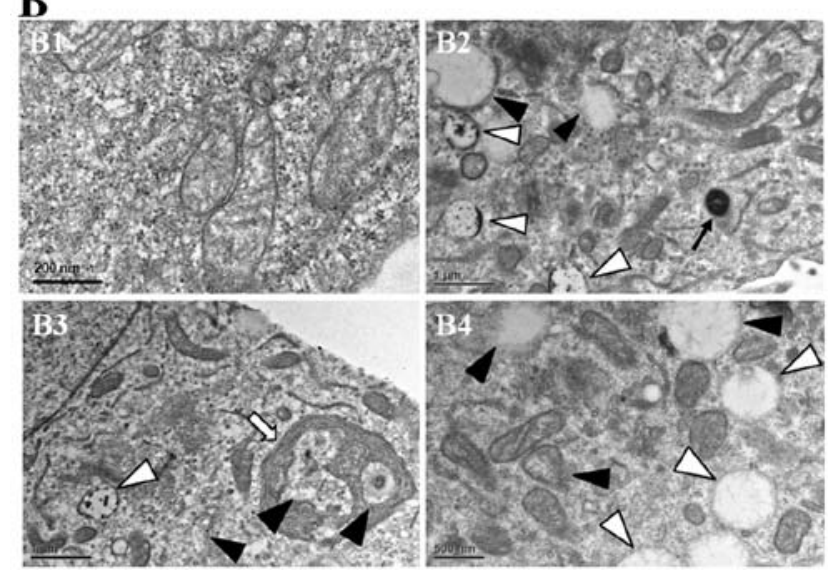

Figure 4. The effect of resveratrol on ATAD3A expression in SKG-I cells (A) Resveratrol reduced ATAD3A expression in a dose-dependent fashion. (B) Effect of ATAD3A expression on ultrastructural changes. Compared to (B1) the wild-type cells, (B2) the evident appearance of autophagic vacuoles (black arrowheads) and degenerated mitochondria or silhouette of mitochondria (white arrow heads) were frequently detected in ATAD3A ${ }^{\text {kd }}$ cells. Smal vesicles in the cytoplasm became more evident, and, occasionally, peroxisomes (black arrow) were identified in ATAD3A ${ }^{\mathrm{kd}}$ cells. (B3) In some cases, autophagosomes (white arrow), containing degenerated mitochondria (black arrowheads), were observed. (B4) In resveratrol-treated cells, autophagic vacuoles (black arrowheads) and degenerated mitochondria as well as silhouettes of mitochondria (white arrow heads) were frequently detected. Small vesicles in the cytoplasm also became more evident. However, unlike in ATAD3A ${ }^{\mathrm{kd}}$ cells, no peroxisomes were identified in resveratrol-treated cells.

Among four HPV-positive squamous cell carcinoma cell lines, three had high levels of ATAD3A expression. Based on the ATAD3A levels in the different cell types, we chose SKG-I and SKG-II cells for the cytotoxicity study.

Our previous study showed that SKG-I was significantly more resistant to anticancer drugs than SKG-II, which was attributed to the presence of DDH (6). However, DDH does not directly inactivate anticancer chemotherapeutics $(14,15)$, suggesting that the desensitization effect on anticancer drugs may involve other factors. To investigate the role of ATAD3A in drug resistance, we silenced ATAD3A (ATAD3A ${ }^{\mathrm{kd}}$ ) expression using an siRNA method. As shown in Fig. 3B, after treatment with siRNA-lentivirus, ATAD3A expression was decreased around 10-fold in SKG-I cells. Drug resistance to cisplatin, ifosfamide, cyclophosphamide, cytarabine, gemcitabine, 5-fluorouracil, docetaxel, paclitaxel, epirubicine, vincristine and doxorubicin markedly decreased in these cells (Fig. 3C). The difference was significant $(\mathrm{p}<0.001)$ in each of the above comparisons. Interestingly, using specific shRNA to silence E6 and E7 (HPV E6/7 $/{ }^{\mathrm{kd}}$ ) expression reduced the protein levels of ATAD3A and $\beta$-actin (Fig. 3D) as well as increased cell apoptosis and cisplatin sensitivity (Fig. 3E). Silencing of E6 and E7 was determined by RT-PCR (24).
Resveratrol inhibition of ATAD3A expression and induction of autophagy and apoptosis. Interestingly, addition of resveratrol reduced ATAD3A expression (Fig. 4A). Because silencing of ATAD3A induced apoptosis and autophagocytosis (18), we therefore used electron microscopy to examine the ultrastructural differences between resveratrol-treated and ATAD3 $\mathrm{A}^{\mathrm{kd}}$ cells. Compared to the control cells (Fig. 4B1), numbers of autophagosome and cytoplasmic vesicles evidently increased in ATAD3A ${ }^{\mathrm{kd}}$ (Fig. 4B2 and B3), and in resveratrol-treated cells (Fig. 4B4). So were mitochondria with defected outer membranes and crumpled matrices. These data correspond well with our previous results that ATAD3A increased drug resistance of cancer cells, possibly via maintaining the appropriate membrane flow and the mitochondrial integrity $(18,25)$. The results suggest that resveratrol may inhibit the trafficking of membrane phospholipids and proteins. Interruption of the process could in some way initiate autophagy and the subsequent apoptosis.

\section{Discussion}

The anti-autophagic ability and facilitation of cancer development of ATAD3A have been shown to be via protection of mitochondrial integrity and prevention of cellular autophagy (18). In this study, we further demonstrated that ATAD3A expression was closely associated with hrHPV infection, validating our previous observations that hrHPV infection was not only critical for carcinogenesis, but also for the disease progression. The current results showed that using PCR and an Easychip HPV blot assay could simultaneously identify the types of HPV infection (6-8). However, using in situ hybridization could adequately locate the HPV infection in UCC, not in the neighboring tissues (6). Moreover, our results indicate that HPV infection correlated with ATAD3A levels as well as those of c-MET, IL-8 and Ki-67 as well as with FIGO stages, lymph node involvement and patients' survival, which corresponded well with our prior studies that expression of these genes might be orchestrated with one another to form a malicious cycle in favor of cancer progression $(6,26,27)$. It is worth noting that, Chinese UCC patients had a higher tendency of overexpressing ATAD3A than the American patients. Interestingly, silencing of ATAD3A expression markedly increased SKG-I cell sensitivity to cisplatin, ifosfamide, cyclophosphamide, cytarabine, gemcitabine, 5-fluorouracil, docetane, paclitaxel, epirubicine, vincristine and doxorubicine. Knockdown of HPV 16 E6/E7 in SKG-I cells, furthermore, reduced protein levels of ATAD3A and $\beta$-actin as well as cisplatin resistance, confirming that E6 and E7 of oncogenic HPV were upstream regulators of survival-related gene expression in UCC cells (28-30). Taken together, our current data indicate that drug resistance in cancer cells, in addition to elevated nuclear DNA repair and cytoplasmic detoxification activities (14-16,31), could be enforced by proteins, such as ATAD3A, which was essential for the maintenance of mitochondrial membrane integrity $(18,19)$.

As noted above, ATAD3A is an ATPase which is involved in an alternative transport of proteins and phospholipids from the endoplasmic reticulum (ER), mitochondria-associated membrane to the mitochondria (18). Silencing of the ATAD3A expression not only altered mitochondrial morphology, but also induced autophagy (18). However, serum starvation increased 
both protein and mRNA levels of ATAD3A in cancer cells, suggesting that a cell self-defense mechanism to conserve energy consumption, while to sustain cell survival was undertaken to avoid a drastic initiation of autophagy $(32,33)$. During initiation of autophagy, the C-terminal 5 amino acids are removed from the microtubule-associated protein light chain 3 (LC-3) to form a LC-3I, which is then conjugated to phosphotidylethanolamine (PE) to form LC-3II, the known marker of autophagy (34). However, the site for LC-3 conjugation to PE, as well as the formation of phagophores, the energy source for the aggregation of autophagosomes and the fusion of autophagosomes with lysosomes is yet to be characterized (32-34). Hailey et al have shown that conjugation of LC-3I to PE could take place on the mitochondria, where the phosphotidylserine (PS) was converted to PE, and where the prospective phagophore, a precursor of the autophagosome, was formed during serum starvation (35). That human mitochondrial fission protein 1 (hFis1) and mitofusin 2 (Mfn2) were detected on both mitochondria and autophagosomes and deletion of the $M f n 2$ gene inhibited the formation of autophagosomes suggesting that hFis1 was required for phagophore formation and that Mfn2, in addition to mitochondrial fusion and tethering between the ER and the mitochondria (36), was required for the fusion of microphagosomes into autophagosomes.

By showing that hypoxia-induced autophagic vesicles were encircled by DRP1 and the human homolog of yeast Rad23 protein A (hHR23A) as well, our previous data suggest that DRP1 and hHR23A may play a role in mammalian cell autophagy (17), based on the fact that hFis1 required DRP1 to carry out fission activities (37). Moreover, our current results provide a reasonable interpretation for the involvement of ATAD3A in autophagy and apoptosis, because ATAD3A may be able to provide energy for the movement of transport vesicles, transporting phospholipids and proteins from the ER, and mitochondria-associated membranes to the mitochondria. Inhibition of ATAD3A function would surely cause a short supply of energy required for transporting phospholipids and proteins to the mitochondria, and membrane abrasions of mitochondria. The loss of mitochondrial membrane integrity would then lead to a failure to keep programmed cell death-related proteins, e.g., apoptosis-inducing factor (AIF) and cytochrome $\mathrm{c}$, in check within the mitochondria. This concept was strengthened by the observations that resveratrol inhibited ATAD3A expression and induced autophagy as well as apoptosis, which corresponded well with results reported by Hsu et al (38).

In conclusion, our current results showed that ATAD3A expression was frequently detected in hrHPV-positive UCC specimens and was correlated with FIGO stages, lymph node involvement and patients' survival. The HPV infection may stabilize ATAD3A expression to prevent initiation of cell autophagy and the subsequent cell apoptosis, hence to increase drug resistance in UCC cells.

\section{Acknowledgements}

RNAi for silencing ATAD3A gene expression was obtained from the National RNAi Core Facility in the Institute of Molecular Biology/Genomic Research Center, Academia Sinica, Taipei, Taiwan, supported by the National Research Program for Genomic Medicine Grants of NSC (NSC
97-3112-B-001-016). This study was supported, in part, by the Comprehensive Academic Promotion Projects (NCHU 995002 to K.C.C.).

\section{References}

1. Annual Reports of the Department of Health, Taiwan, 2008.

2. Walboomers JM, Jacobs MV, Manos MM, et al: Human papillomavirus is a necessary cause of invasive cervical cancer worldwide. J Pathol 189: 12-19, 1999.

3. zur Hausen H: Papillomaviruses in anogenital cancer as a model to understand the role of viruses in human cancers. Cancer Res 49: 4677-4681, 1989.

4. Stoler MH: Human papillomaviruses and cervical neoplasia: a model for carcinogenesis. Int J Gynecol Pathol 19: 16-28, 2000.

5. Chan SY, Delius H, Halpern AL and Bernard HU: Analysis of genomic sequences of 95 papillomavirus types: uniting typing, phylogeny, and taxonomy. J Virol 69: 3074-3083, 1995.

6. Ueda M, Hung YC, Chen JT, et al: Infection of human papillomavirus and overexpression of dihydrodiol dehydrogenase in uterine cervical cancer. Gynecol Oncol 102: 173-181, 2006.

7. Lai CH, Huang HJ, Hsueh S, et al: Human papillomavirus genotype in cervical cancer: a population-based study. Int J Cancer 120: 1999-2006, 2007.

8. Lai CH, Chang CJ, Huang HJ, et al: Role of human papillomavirus genotype in prognosis of early-stage cervical cancer undergoing primary surgery. J Clin Oncol 25: 3628-3634, 2007.

9. Ding DC, Hsu HC, Huang RL, et al: Type-specific distribution of HPV along the full spectrum of cervical carcinogenesis in Taiwan: an indication of viral oncogenic potential. Eur J Obstet Gynecol Reprod Biol 140: 245-251, 2008.

10. Scheffner M, Munger K, Byrne JC and Howley PM: The state of the p53 and retinoblastoma genes in human cervical carcinoma cell lines. Proc Natl Acad Sci USA 88: 5523-5527, 1991.

11. Nagai Y, Toma T, Moromizato H, et al: Persistence of human papillomavirus infection as a predictor for recurrence in carcinoma of the cervix after radiotherapy. Am J Obstet Gynecol 191: 1907-1913, 2004.

12. Im SS, Wilczynski SP, Burger RA and Monk BJ: Early stage cervical cancers containing human papillomavirus type $18 \mathrm{DNA}$ have more nodal metastasis and deeper stromal invasion. Clin Cancer Res 9: 4145-4150, 2003.

13. Yokoyama M, Iwasaka T, Nagata C, et al: Prognostic factors associated with the clinical outcome of cervical intraepithelial neoplasia: a cohort study in Japan. Cancer Lett 192: 171-179, 2003.

14. Hsu NY, Ho HC, Chow KC, et al: Overexpression of dihydrodiol dehydrogenase as a prognostic marker of non-small cell lung cancer. Cancer Res 61: 2727-2731, 2001.

15. Deng HB, Parekh HK, Chow KC and Simpkins H: Increased expression of dihydrodiol dehydrogenase induces resistance to cisplatin in human ovarian carcinoma cells. J Biol Chem 277: 15035-15043, 2002.

16. Chen YJ, Yuan CC, Chow KC, et al: Overexpression of dihydrodiol dehydrogenase is associated with cisplatin-based chemotherapy resistance in ovarian cancer patients. Gynecol Oncol 97: 110-117, 2005.

17. Chiang YY, Chen SL, Hsiao YT, et al: Nuclear expression of dynamin-related protein 1 in lung adenocarcinomas. Mod Pathol 22: 1139-1150, 2009.

18. Fang HY, Chang CL, Hsu SH, et al: ATPase family AAA domain-containing $3 \mathrm{~A}$ is a novel anti-apoptotic factor in lung adenocarcinoma cells. J Cell Sci 123: 1171-1180, 2010.

19. Kuzuya K: Chemoradiotherapy for uterine cancer: current status and perspectives. Int J Clin Oncol 9: 458-470, 2004.

20. Ishiyama M, Tominaga H, Shiga M, Sasamoto K, Ohkura $Y$ and Ueno K: A combined assay of cell viability and in vitro cytotoxicity with a highly water-soluble tetrazolium salt, neutral red and crystal violet. Biol Pharm Bull 19: 1518-1520, 1996.

21. Remmele W and Schicketanz KH: Immunohistochemical determination of estrogen and progesterone receptor content in human breast cancer. Computer-assisted image analysis (QIC score) vs. subjective grading (IRS). Pathol Res Pract 189: 862-866, 1993.

22. Kaplan EL and Meier P: Nonparametric estimation from incomplete observations. J Am Stat Assoc 53: 457-481, 1958.

23. Mantel N: Evaluation of survival data and two new rank order statistics arising in its consideration. Cancer Chemother Rep 50: 163-170, 1966. 
24. Qi Z, Xu X, Zhang B, et al: Effect of simultaneous silencing of HPV-18 E6 and E7 on inducing apoptosis in HeLa cells. Biochem Cell Biol 88: 697-704, 2010.

25. Huang KH, Chow KC, Chang HW, Lin TY and Lee MC: The ATPase family, AAA domain containing $3 \mathrm{~A}$ is an anti-apoptotic factor and a secretion regulator of PSA in the prostate cancer. Int J Mol Med 28: 9-15, 2011.

26. Chen JT, Huang CY, Chiang YY, et al: HGF increases cisplatin resistance via down-regulation of AIF in lung cancer cells. Am J Respir Cell Mol Biol 38: 559-565, 2008.

27. Chiang YY: Hepatocyte growth factor induces hypoxia-related interleukin-8 expression in lung adenocarcinoma cells. Mol Carcinog 48: 662-670, 2009.

28. Wang X, Meyers C, Guo M and Zheng ZM: Up-regulation of p18Ink4c expression by oncogenic HPV E6 via p53-miR-34a pathway. Int J Cancer: Dec 2, 2010 (Epub ahead of print).

29. McCloskey R, Menges C, Friedman A, Patel D and McCance DJ: Human papillomavirus type 16 E6/E7 upregulation of nucleophosmin is important for proliferation and inhibition of differentiation. J Virol 84: 5131-5139, 2010

30. Au Yeung CL, Tsang WP, Tsang TY, Co NN, Yau PL and Kwok TT: HPV-16 E6 upregulation of DNMT1 through repression of tumor suppressor p53. Oncol Rep 24: 1599-1604, 2010.
31. Lai TC, Chow KC, Lin TY, et al: Expression of 53BP1 as a cisplatin-resistant marker in patients with lung adenocarcinomas. Oncol Rep 24: 321-328, 2010.

32. Shintani T and Klionsky DJ: Autophagy in health and disease: a double-edged sword. Science 306: 990-995, 2004.

33. Yang Z and Klionsky DJ: Eaten alive: a history of macroautophagy. Nat Cell Biol 12: 814-822, 2010.

34. Taylor MP and Kirkegaard K: Modification of cellular autophagy protein LC3 by poliovirus. J Virol 81: 12543-12553, 2007.

35. Hailey DW, Rambold AS, Satpute-Krishnan P, et al: Mitochondria supply membranes for autophagosome biogenesis during starvation. Cell 141: 656-667, 2010.

36. de Brito OM and Scorrano L: Mitofusin 2 tethers endoplasmic reticulum to mitochondria. Nature 456: 605-610, 2008.

37. Lee YJ, Jeong SY, Karbowski M, Smith CL and Youle RJ: Roles of the mammalian mitochondrial fission and fusion mediators Fis1, Drp1, and Opa1 in apoptosis. Mol Biol Cell 15: 5001-5011, 2004.

38. Hsu KF, Wu CL, Huang SC, et al: Cathepsin L mediates resveratrolinduced autophagy and apoptotic cell death in cervical cancer cells. Autophagy 5: 451-460, 2009. 